\title{
Implementation Results of a Novel Comprehensive Mental Skills Curriculum during Simulator Training
}

Dimitrios Stefanidis MD, PhD, ${ }^{1,2}$ Nicholas Anton, ${ }^{2}$ Graham McRary, ${ }^{2}$ Lisa D Howley $\mathrm{PhD},{ }^{2,3}$ Manuel Pimentel, ${ }^{2}$ Cameron Davis, ${ }^{3}$ Ashley M Yurco, ${ }^{3}$ Nick Sevdalis, PhD,${ }^{4}$ Charles Brown, $\mathrm{PhD}{ }^{5}$

1 Department of Surgery, Carolinas HealthCare System, Charlotte, NC

2 Carolinas Simulation Center, Carolinas HealthCare System, Charlotte, NC

3 Medical Education, Carolinas HealthCare System, Charlotte, NC

4 King's College, London, UK

5 Head in the Game, Inc, Charlotte, NC

Correspondence concerning this article should be addressed to:

Dimitrios Stefanidis, MD, PhD, FACS, FASMBS

Surgical and Research Director, Carolinas Simulation Center

Associate Professor of Surgery, Carolinas Healthcare System

Clinical Associate Professor of Surgery, University of North Carolina

Division of Bariatric Surgery, Department of Surgery

Carolinas HealthCare System

1025 Morehead Medical Dr. Suite 300

Charlotte, NC 28204

Email: dimitrios.stefanidis@ carolinashealthcare.org

Phone: (704) 355-5114

\section{Keywords}

Surgery, Simulation Training, Stress, Mental Skills, Stress Management, Surgical Training, Performance Enhancement

This study was presented from the podium at the annual meeting of the Association for Surgical Education in Seattle, WA on April 24 ${ }^{\text {th }}, 2015$.

Acknowledgement

Financial support for this study was provided by the Agency of Healthcare Research and Quality (AHRQ) \# R18HS022080. Sevdalis' research was funded by the National Institute for Health Research (NIHR) Collaboration for Leadership in Applied Health Research and Care South London at King's College Hospital NHS Foundation Trust. The views expressed are those of the authors and not necessarily those of the NHS, the NIHR or the Department of Health.

This is the author's manuscript of the article published in final edited form as:

Stefanidis, D., Anton, N. E., McRary, G., Howley, L. D., Pimentel, M., Davis, C., ... Brown, C. (2017).

Implementation results of a novel comprehensive mental skills curriculum during simulator training. The American Journal of Surgery, 213(2), 353-361. https://doi.org/10.1016/j.amjsurg.2016.06.027 


\begin{abstract}
Introduction

Mental skills training refers to the implementation of cognitive performance-enhancing strategies to promote optimal performance. We aimed to develop a surgery-specific mental skills curriculum (MSC) and obtain initial evidence of efficacy.
\end{abstract}

\title{
Methods
}

The developed MSC consisted of eight proven performance-enhancing modules. Its efficacy was assessed during laparoscopic simulator-based practice by novices using validated instruments of mental skills, workload, and stress, in addition to a skill transfer test to a porcine model. A paired t-test was used to analyze the data.

\section{Results}

Nine surgical novices completed the curriculum. Compared with baseline assessment participants improved significantly their laparoscopic performance and mental skills after completion of the MSC. All participants completed the task in the porcine model without an appreciable change in their perceived stress. During the skill transfer test, eight participants were observed using mental skills taught in the MSC.

\section{Conclusions}

A surgery-specific simulator-based mental skills curriculum was developed and its efficacy in improving mental skills and surgical performance was supported during a surgical skill transfer test. 


\section{Background}

Working as a surgeon not only requires extensive medical knowledge, critical thinking skills, and fine motor control, but also endurance and stamina, both physical and psychological. Surgeons must be able to maintain attention, make split-second decisions, and retain fine motor control throughout the entirety of a surgical procedure, sometimes lasting several hours. In addition to operating, surgeons must also effectively lead and communicate with their operating room team. The complexities, implications, and risks associated with operations create a generally stressful environment. ${ }^{1-2}$ Further, advances in surgery continue to increase the demands on surgeons. For example, laparoscopic surgery is notably more difficult and stressful than traditional open surgery. ${ }^{3}$ Increased intraoperative stress may jeopardize patient safety by negatively affecting surgeons' coordination, emotional state, concentration, and decision making ability. ${ }^{4}$ Novice surgeons, compared to expert surgeons, are more likely to experience the negative effects of stress according to objective (e.g., heart rate variability and heart rate) and subjective (e.g., self-report) measures of stress. ${ }^{1}$ In novice laparoscopic surgeons, stress has been shown to negatively impact economy of motion, amount of time taken, and errors made. ${ }^{5}$

Results from a survey of 72 surgeons from Carolinas Medical Center showed that "complex or rarely performed cases" were perceived as the most stressful intraoperative factors, whereas "personal life distractions" and "multitasking requirements" as the least stressful. In general, trainees perceived greater amounts of stress during surgery than attending physicians. Importantly, nearly $40 \%$ of respondents indicated that they had witnessed an intraoperative complication due to stress and $82 \%$ felt that stress management training would be beneficial. ${ }^{6}$ Similar findings have been reported internationally. ${ }^{4}$ 
Mental skills are trainable mental abilities that support successful learning and performance, ${ }^{7}$ with the aim of consistently reaching the user's upper range of ability. In sport psychology mental skills have been widely successful in helping athletes consistently perform at high levels. ${ }^{8}$ Furthermore, mental skills interventions have been shown to improve performance with US Navy SEALS, ${ }^{9}$ military pilots, ${ }^{10}$ police special forces, ${ }^{11}$ and elite athletes. ${ }^{12}$ Not only are mental skills curricula designed to decrease the effects of stress, but they can also help with mental imagery (practice or rehearsal), arousal regulation skills, attention management skills, goal-setting, and performance-enhancing pre-performance routines. ${ }^{13-17}$

Research has shown that mental skills optimization can also benefit surgical performance and stress reduction in the operating room. ${ }^{18-19}$ Mental imagery has been demonstrated to enhance surgeons' performance, skill acquisition, ${ }^{20-21}$ knowledge, ${ }^{22}$ and confidence. ${ }^{22}$ Arousal regulation skills have also been effective at reducing surgeons' psychological and physiological stress. ${ }^{18-19}$ Interviews with expert surgeons conducted at our institution indicate that they regularly use mental skills to enhance their performances and reduce levels of stress (unpublished data). Therefore, we hypothesize that the implementation of a mental skills curriculum (MSC) will aid in the reduction of perceived stress and workload and improve surgical performance.

The objective of this study was to develop a surgery-specific MSC to be implemented during simulation training and obtain initial evidence of efficacy in improving performance. We specifically aimed to demonstrate improvements in mental skills and laparoscopic performance after training.

\section{Methods}


A novel MSC was developed by an interdisciplinary team consisting of a surgeon educator with expertise in laparoscopic surgery and simulation-based research, a performance psychologist with expertise in mental skills training, and a $\mathrm{PhD}$ educator with expertise in instructional design. Through adaptation of David Kern's (2009) model, the curriculum was devised to include a needs assessment, development of goals and instructional objectives based on this assessment, identification and development of instructional methods, and development of assessment and evaluation methods for the measurement of curriculum outcomes. ${ }^{23}$ The developed curriculum consisted of eight modules: introduction to mental skills, mental imagery, goal setting, energy management (relaxation), attention management, refocusing strategies, and performance routines (see Table 1). The modules for this curriculum were chosen based on empirical work on the implementation of comprehensive mental skills curricula implemented in the military, sport, individual mental skill interventions implemented with surgeons, and the expertise of the collaborating performance psychologist with 35 years of consulting experience in mental skills training of high performers in many disciplines, including medicine.

In an Institutional Review Board-approved study, a convenience sample of surgical novices ( $n=9)$ including pre-medical college and medical students, were enrolled to study the effectiveness of the MSC. We aimed to include 10 participants and initially recruited 15, but because the study ran over the summer, four of them could not finish as they had to leave the city, and two were unable to finish due to previous commitments. Participants were volunteers and gave informed consent prior to their participation. After a baseline assessment of laparoscopic and mental performance, participants completed the MSC and trained using laparoscopic simulators over the course of an eight week period. Their performance was again evaluated after curriculum 
completion and compared to baseline. In addition, a transfer test using a porcine model was performed to assess the effectiveness of training

\section{Baseline Assessment}

Baseline assessment consisted of a questionnaire completed by all participants, which included demographic items, and items measuring prior laparoscopic and simulator experience. A baseline measurement of participants' heart rates was also recorded with a Polar FS2 heart rate monitor (Polar Electro Inc., Lake Success, NY). In addition, the modified Test of Performance Strategies (TOPS-2), the d2 Test of Attention (d2), the State-Trait Anxiety Inventory (STAI), and the Mental Imagery Questionnaire (MIQ) were completed by participants to assess mental abilities. Participants then watched introductory videos to the FLS PEG Transfer and intracorporeal suturing tasks. Their performance on those tasks (time taken to complete a repetition and errors) was recorded. Upon completing the tasks, the participants completed the NASA-TLX workload assessment.

The Test of Performance Strategies Version 2 (TOPS-2) is a 68-item self-report instrument designed to measure a comprehensive range of psychological skills and techniques which have been shown to impact successful athletic performance. These skills and techniques include goal-setting, relaxation, activation, imagery, self-talk, emotional control, and automaticity. ${ }^{26}$ The instrument enables measurements of the frequency of participants' use of these seven skills in both practice and competition. Example items from the TOPS-2 include: "I set realistic but challenging goals for practice", "I rehearse my performance in my mind before practice", and "I talk positively to myself to get the most out of my performance during a procedure". Participants rate the frequency with which the implement these skills using a 5-point Likert scale $(1=$ never $-5=$ always $)$. The TOPS- 2 has been shown to discriminate between elite 
and high-level performers in non-competition performance settings. Furthermore, there is strong evidence supporting its reliability and validity. ${ }^{26}$ The non-normed fit index (NNFI) and comparative fit index $(\mathrm{CFI})$ are excellent for both the competition and practice subscales, $(0.97$ and 0.97 , and 0.96 and 0.96 , respectively). ${ }^{26}$ This instrument was modified with permission to better suit its use with surgeons.

The $\mathrm{d} 2$ Test of Attention is a written, timed test of selective attention. The items are composed of the letters "d" and "p" with one, two, three, or four dashes arranged either individually or in pairs above and below the letter (see Figure 1). The user is given 20 seconds to scan each line and mark all "d's" with two dashes. There are 14 lines of 47 characters each for a total of 658 items. Measures of performance include total number of items processed (TN), percentage of errors $(\mathrm{E} \%)$, an index of concentration performance $(\mathrm{CP}) \mathrm{TN}$ - errors $(\mathrm{TN}-\mathrm{E})$, and fluctuation rate across trials (FR). ${ }^{27}$

The STAI is a 40-item self-report questionnaire that uses the 4-point Likert scale and measures state anxiety, otherwise known as anxiety about an event, and trait anxiety, or anxiety as a personal characteristic. ${ }^{28}$ Example state anxiety items from the STAI-full measure include: "I feel calm" and "I am tense". Trait anxiety items include: "I feel nervous and restless" and "I worry too much about something that doesn't really matter". The shortened version of STAI, the STAI-6, consisting of only six questions, focuses on state anxiety only and has been shown to have internal consistency, reliability, and validity. ${ }^{29}$ The full version of the STAI was used at baseline and post-training assessment sessions, and the STAI-6 was used prior to, during, and following participants' completion of FLS tasks (at baseline, training, and post-training) and the surgical skill transfer test. 
The MIQ is a validated measure of the quality and volume of a surgeon's mental imagery during performance of a laparoscopic cholecystectomy. All dimensions of mental imagery that are related to surgery are assessed, including visual and kinesthetic imagery, confidence to carry out the procedure, and perceived usefulness of engaging in imagery preoperatively. Example items from the MIQ include: "How easily can you 'see' yourself performing a Laparoscopic task/procedure?" and "How vivid and clear are the images of a Laparoscopic task/procedure in your mind?" Responses range on a 7-point Likert scale $(1=$ Not at all $-7=$ Very. The MIQ has been validated for use with surgeons and shown to adequately capture mental imagery quality of both junior and senior surgeons. ${ }^{30}$

The NASA-TLX is an assessment used to provide subjective measurement of perceived workload during performance of a task. Participants rate their mental demand, physical demand, temporal demand, performance, effort, and frustration on a 21-point Likert scale $(1=$ very low to 21 = very high). ${ }^{31}$ Example items from the NASA-TLX include: "How mentally demanding was the task?" and "How hard did you have to work to accomplish your level of performance?"

The FLS peg transfer task, which is designed to train visual-spatial and depth perception on a two-dimensional screen from a monocular viewpoint, is the most basic of the five FLS manual skill tasks. The peg transfer task uses six plastic rings on a board with 12 pegs. In the beginning, all of the rings are on the participant's non-dominant (ND) side. The participants then use grasping forceps to lift the ring off the peg, transfer it in space from their ND to dominant (D) hand $^{32}$, and place the ring on a peg on the $\mathrm{D}$ hand side. After all six rings have been transferred from the ND to D side of the board, the process is reversed, transferring all six rings from the D side to ND side in the same steps. Peg transfer task performance was assessed using the following 
equation: Performance Score = maximum time allowed (300 seconds) - task completion time (seconds) $-[10 \mathrm{x}$ errors (number of rings dropped out of view of the camera) $] .{ }^{15-17}$

The FLS intracorporeal suturing model is a 3-cm Penrose drain with a slit on top and two black targets on both sides of the slit. The Penrose drain is attached in the training box with Velcro. Participants use a 6-inch, 2-0 silk suture with a tapered needled to place one stitch in the Penrose drain to close the slit. The participants are instructed to pass the needle through the two opposite sides adjacent to the slit, aiming for the black targets. To secure the stitch, the participants must first tie a surgeon's knot, followed by two square knots. Participant's suturing performance was assessed using a previously published objective score: Performance Score = maximum time allowed (600 seconds) - task completion time (seconds) - [10 x accuracy error $(\mathrm{mm}$ outside black target $)]-[100 \mathrm{x}$ security error $(\mathrm{slip}=1$ or knot break $=2)] .{ }^{15-17}$

During the FLS peg transfer task and intracorporeal suturing task the participants' heart rates were recorded. After the participants' ten minute testing session, they completed the NASATLX questionnaire.

\section{Training}

After completion of baseline assessments, participants were scheduled to participate in eight, weekly mental skills and FLS training sessions in small groups or individually (depending on participant availability) with the mental skills/FLS coach, who has a background in performance psychology, facilitating all sessions. Each one of the sessions lasted approximately 90 minutes, with the first 45 minutes dedicated to the MSC, and the last 45 minutes used for applied mental skills and FLS Peg Transfer and FLS IS training. The MSC curriculum provided participants with the eight video education modules and an accompanying workbook. Each week a different module was taught. The MSC was conducted in a small classroom and consisted of individual or 
small group (up to three participants) sessions based on participant availability. Participants were instructed on how to optimally apply the aforementioned mental skills. Immediately following completion of the module, the participants were asked to apply what they learned to their FLS simulation training to help solidify the mental skills. For example, during the energy management module, participants were encouraged to consider methods of integrating centered breathing to control physiological arousal before and during FLS training, in aims of integrating this skill as a habitual part of their performance. The goal for participants' performance was based on previously published, expert-derived performance goals. During the first four sessions, participants trained on the peg transfer task, which had a performance goal of 48 seconds without errors. During the last four sessions the participants trained on intracorporeal suturing, aiming for a goal of 112 seconds with no errors of accuracy or knot security on two consecutive repetitions and ten additional repetitions. ${ }^{32}$

Following the eight-week training period, participants were retested using the same measures and procedures as those used in the baseline assessment. 


\section{Transfer Test}

Participants also performed laparoscopic suturing on a live porcine fundoplication model to demonstrate the transfer of their simulator-acquired skills. Regardless of performance during the training sessions, participants were allowed to participate in the porcine transfer test. The transfer test consisted of participants placing three gastrogastric sutures on a live, anesthetized porcine model. An expert laparoscopic surgeon prepared the porcine model by placing trocars, and four blue colored stitches on the gastric fundus to serve as target sites for the suturing task. Participants were instructed to place three sutures: two sutures to connect the preplaced marking stitches and a third suture between the first two. Each successful suture required a surgeon's knot and two square knots to be placed. In addition, to be considered successful, each suture must be placed in a maximum of ten minutes. All participant created knots were assessed by the expert laparoscopic surgeon to determine accuracy and knot security after their completion. Knots were assessed using the same objective scoring method as for the FLS IS task.

Participants' heart rates were monitored continuously throughout the testing session. Following the completion of the testing session, participants completed a NASA-TLX questionnaire.

Analyses

To assess the efficacy of the novel Mental Skills Curriculum, participants' baseline performance scores were compared to their post-training assessment scores. Paired t-tests were used to analyze the data and a $p$-value of $\mathrm{p}<0.05$ was considered statistically significant. To simplify comparisons among test results, scores were normalized to baseline performance: Normalized Score $=$ Actual Score/ Baseline Score. Heart rate was also normalized to baseline resting heart rate. Feedback 
from the participants regarding the study and curriculum were sought in the form of open-ended responses.

\section{Results}

Nine surgical novices (age $23 \pm 7$ years, $55 \%$ women, $78 \%$ right handed) completed the curriculum. At the conclusion of training, participants had completed $49 \pm 23$ peg transfer repetitions and $21 \pm 9$ intracorporeal suturing repetitions during practice.

In comparison with baseline, participants significantly improved their laparoscopic performance and mental skills after completing training (see Figure 2). The FLS peg transfer and intracorporeal suturing scores increased significantly from baseline to post-training $(11 \pm 33 \mathrm{vs}$ $183 \pm 62, \mathrm{p}<0.0001$ and $13 \pm 39$ to $328 \pm 202, \mathrm{p}<0.01$, respectively). All participants were able to complete the porcine suturing task, and as a group, achieved a score of $244 \pm 143$, which reflects a $74 \%$ skill transfer compared to their performance during the simulator post-test assessment. Significant improvements from baseline to the post-test session were found in the mTOPS-2 assessment ( $219 \pm 18$ vs $263 \pm 23, \mathrm{p}<0.01)$, d2 (538 \pm 64 vs $595 \pm 60, \mathrm{p}<0.001)$, and MIQ $(3.8 \pm 2.3$ vs $5.8 \pm 0.7, \mathrm{p}<0.001)$.

No significant differences were observed in the average participant heart rate between the baseline and post-test session while resting $(74 \pm 10$ vs $79 \pm 11$, $p=$ not significant, respectively), or while performing intracorporeal suturing ( $88 \pm 7$ vs $95 \pm 11$; $p=$ not significant, respectively). Participant heart rate during the porcine session significantly increased from baseline testing to $102 \pm 18$ at rest $(\mathrm{p}<0.01)$, and $119 \pm 16$ during testing $(\mathrm{p}<0.01)$ (see Figure 3$)$. Porcine session heart rate recordings were also significantly higher than during the post-test session (102 \pm 18 vs $79 \pm 11$, $\mathrm{p}<0.02$, respectively at rest and $119 \pm 16$ vs $95 \pm 11, \mathrm{p}<0.02$, respectively during testing). 
There was a decrease in NASA-TLX score from $20 \pm 2$ at baseline to $15 \pm 4$ during the posttest $(\mathrm{p}<0.01)$ and to $17 \pm 3$ during the transfer test $(\mathrm{p}<0.05)$. The NASA-TLX score increased significantly from the post-test to the porcine session $(15 \pm 4$ vs $17 \pm 3, \mathrm{p}<0.05$, respectively). Similarly, participants' STAI-6 scores decreased significantly from baseline to the post-test session ( $15 \pm 3$ vs $12 \pm 3, \mathrm{p}<0.05$, respectively) but did not change significantly between baseline and porcine testing ( $15 \pm 3$ vs $16 \pm 2$, p=not significant, respectively). The STAI- 6 scores also increased significantly from the post-test session to the porcine session $(12 \pm 3$ vs $16 \pm 2, p<0.002$, respectively).

Eight out of the nine participants were observed to use mental skills (deep breathing and trigger breaths that were taught during the MSC) during the porcine test. Participants reported that training improved their mental skills, and was very valuable to enhancing their performance. One participant stated that:

"being able to calm myself by lowering the heart rate with centered breathing has been very helpful and I have seen great improvement in performance. When I know I have a large procedure or exam, I usually get anxious and my heart rate increases rapidly as the time of action approaches, but this approach has helped me reduce physical shakiness and anxiety during a performance."

Similarly, another participant noted that:"I was able to specifically practice developing an action plan for peg transfer, suture practice, and porcine lab through the workbook exercises based on examples shown in the workbook."

\section{Discussion}


This paper reports the development of a novel, surgery-specific, comprehensive mental skills curriculum aimed at enhancing surgical performance and provide preliminary evidence of its effectiveness. It was found that by using this novel curriculum during simulator training, participants not only improved their laparoscopic performance but also demonstrated significant improvements in mental abilities after training based on a battery of validated assessment tools. The improvement in the modified mTOPS-3 scores after training is indicative that the MSC was effective in teaching a comprehensive range of psychological skills and techniques that can be applied to surgical performance. The improvements seen in $\mathrm{d} 2$ scores suggest that participants were better able to maintain attention on the task and concentrate at higher levels than prior to MSC training. The enhanced MIQ scores after training suggest that the quality and volume of the participant's mental imagery, including pre-task routine imagery and confidence to carry out the task significantly improved compared with baseline. Significant increases in participant heart rate were documented during the porcine session (approximately 40 beats per minute higher than at baseline), which is indicative of physiological stress related to the operating room environment, which novices had not been exposed to before, and to performance anxiety as demonstrated in previous studies. ${ }^{17}$ However, despite this notable increase in physiological stress, the participants' perception of their stress, anxiety, and workload, as indicated by STAI-6 and NASA-TLX selfreports, were not significantly increased from baseline testing. Thus, while the MSC curriculum does not appear to have affected physiological stress, it seems to have had an effect on perceived stress and workload, as participants did not appear to experience increased stress and workload compared to their baseline. Indeed, Hardy's Catastrophe Model of performance indicates that there are two primary dimensions to performance stress: a physiological dimension and a cognitive dimension. ${ }^{33}$ Of the two dimensions, cognitive anxiety/stress is more detrimental to 
performance. When a performer is able to manage the cognitive/emotional dimension, the increase in physiology can actually be associated with heightened physiological arousal of one's ideal performance state. ${ }^{33}$ The noted improvements in mental skills may also have influenced the dramatic improvement in laparoscopic performance and the transfer of the acquired skill to the clinical environment. If MSC training allows participants to be able to better prepare for tasks, maintain focus, and manage anxiety and stress effectively, then the implications for performance and surgical training are apparent. For surgeons who operate without room for mistakes and under elevated stress levels, the implementation of an effective MSC could directly positively impact their performance, and in turn, patient outcomes.

Mental imagery and stress management techniques, which usually include relaxation skills and imagery, have been demonstrated to be beneficial for surgeons in previous studies. ${ }^{18-22}$ Immenroth et al. (2007) showed that surgeons who received imagery training performed simulated laparoscopic cholecystectomies significantly better than those who did not. ${ }^{21}$ Arora et al. (2010) found that both novices (having performed <10 laparoscopic cholecystectomies) and experienced surgeons (having performed $>100$ laparoscopic cholecystectomies), benefited by mental imagery training. ${ }^{22}$ The same authors found that when mental imagery was implemented with physical practice, surgical novices significantly improved their laparoscopic cholecystectomy performance on a virtual reality (VR) simulator. Furthermore, the learning curve for the experimental group participants was shortened in comparison with the control group. ${ }^{20}$ Arora et al. (2011) also showed that mental imagery effectively reduced surgical novices' stress while performing VR laparoscopic cholecystectomies indicated by State-Trait Anxiety Inventory scale, heart rate, and cortisol levels. ${ }^{18}$ Further, Wetzel et al. (2011) found that when experienced surgeons implemented an imagery and relaxation skill-based stress management program prior to 
a simulated carotid endarterectomy, they had significantly better stress coping skills (measured using the Surgical Coping Questionnaire), teamwork (measured using the Observational Teamwork Assessment for Surgery), and reduced stress (measured using heart rate variability) compared to controls. ${ }^{19}$

There were some methodological differences between the Arora et al. (2011) and Wetzel et al. (2011) studies and our study, which likely explain the observed differences in physiological stress responses. Arora et al. (2011) trained surgical novices but tested them using the same platform they trained in (VR simulator) and not in the clinical environment. It has been previously demonstrated that testing novices in the clinical environment leads to significantly heightened stress, ${ }^{17}$ which was encountered in this study. It is possible that participant stress/performance anxiety were too high for participants to control. On the other hand, participants' perceived stress (e.g., cognitive) did not change despite their significant physiologic arousal. This may indicate participants are able to handle stress muchmore effectively, which could be an effect of the mental skills training. The Wetzel et al. (2011) study was conducted with experienced surgical residents (those $\geq 2$ years in residency). These individuals may have been able to better assimilate mental skills techniques given that they did not also have to learn surgical skills at the same time, unlike our novice participants. Moreover, the performance tests participating surgeons took part in during the Wetzel et al. (2011) study were 2 simulated carotid endarterectomies (CEA) crisis scenarios. Based on the experience level of the residents, they were all able to perform a CEA as the primary surgeon. Thus, the simulated scenarios in Wetzel et al.'s study may have been more familiar to their participants, as they may have encountered similar clinical scenarios previously. Again, the unfamiliar nature of the operating room environment for our participants may have contributed to their physical stress. 
Also, the mental skills curriculum developed in this study includes elements similar to the studies reported above, but is much more comprehensive in its coverage. In addition to mental imagery, the developed curriculum integrates goal setting, attention and activation management, refocusing strategies and pre-performance routines, which are currently used in other disciplines to more comprehensively address mental skills necessary for optimal performance. Indeed, in other disciplines comprehensive mental skills programs have been shown to be more effective than the application of a single technique. ${ }^{8,10,12-14}$ For example, McCrory et al. (2013) introduced a mental skills training program to military pilot trainees in an effort to enhance their confidence to operate the aviation equipment and ability to manage stress. ${ }^{10}$ The program consisted of goal setting, imagery, and attention management mental skills training sessions. At the conclusion of the study, participants displayed significantly increased confidence to operate the aviation equipment and through the use of mental skills, reduced anxiety, and increased self-regulatory behavior (e.g., flight planning, remembering flight brief information, contingency planning, etc.). The comprehensive nature of McCrory et al.'s (2013) training program directly enhanced a number of critical areas of participants' performance, which likely would not have been possible by implementing a single-skill intervention.

\section{Limitations}

This study is subject to the limitations of a small scale pre-post test design and did not include a control group. Given that the objective was to obtain initial experience with the curriculum, document its preliminary effectiveness, and identify areas in need of improvement prior to its optimization and application with a larger group of learners, a convenience sample of nine participants was used. Despite the relatively small sample size, significantly enhanced mental skills after training were observed and enough feedback was obtained allowing us to make 
necessary modifications to optimize the curriculum for the next step in its evaluation process. In addition, while the effectiveness of the curriculum in enhancing mental skills was clearly demonstrated, the observed improvement in participant laparoscopic performance could be a consequence of proficiency-based training rather than mental skills training; in the absence of a control group this question cannot be answered by this study. In order to further investigate the effectiveness of the described MSC on laparoscopic performance, a larger, adequately powered randomized controlled trial including a control group is presently ongoing at our institution to further validate this study's results. In addition, static heart rate readings were recorded as the main physiological determinant of stress rather than heart rate variability. The literature suggests that heart rate variability is a more accurate measure of physiological response to stress. ${ }^{34-35}$ However, in a prior study by the authors, it was found that the participant's static heart rate was a more sensitive gauge of stress than heart rate variability. ${ }^{17}$ Also, due to the comprehensive nature of this curriculum, it's difficult to determine if the observed improvements in mental imagery (assessed with the MIQ), attention (assessed with the d2), or general mental skill use (assessed with the TOPS) after the implementation of our curriculum were the result of just one mental skill strategy or a combination thereof. However, this curriculum was designed for the mental skills strategies to be highly integrated with one another (e.g., refocusing strategies integrates elements of goal setting and action plans, energy and attention management, and mental imagery), with the ultimate goal of enhancing surgical performance and reducing learner stress. Thus, while the effectiveness of individual strategies is important, it is more important to integrate them in such a way that it provides the best possible eventual outcome even at the expense of not knowing for certain which strategy may have contributed the most to that success. A good example in surgery that follows the same concept is the ERAS paradigm or the combination of several strategies 
proven or likely to improve patient outcomes into a comprehensive set of actions that lead to patient care improvements. ${ }^{36}$

\section{Conclusion}

A surgery-specific comprehensive simulator-based mental skills curriculum was developed and its efficacy in improving mental skills and surgical performance demonstrated during a transfer test. Drawing from the reported results, such a curriculum may better prepare surgeons to manage the stress of a surgical environment, improve their performance, and ultimately positively impact patient outcomes. Current and upcoming studies from the authors will help further validate these findings and support their surgical applications. 


\section{References}

1. Arora, S, Sevdalis, N, Nestel, D, et al. The impact of stress on surgical performance: a systematic review of the literature. Surgery 2010a; 147:318-30.

2. Maher, Z, Milner, R, Cripe, J, et al. Stress training for the surgical resident. The American Journal of Surgery 2013; 205:169-74.

3. Berguer, R, Smith, WD, Chung, YH. Performing laparoscopic surgery is significantly more

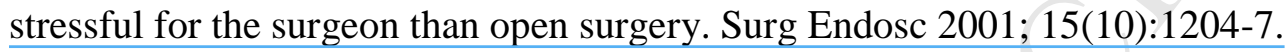

4. Wetzel, CM, Kneebone, RL, Woloshynowych, M, et al. The effects of stress on surgical performance. American Journal of Surgery 2006; 191:5-10.

5. Arora S, Sevdalis N, Aggarwal R, et al. Stress impairs psychomotor performance in novice laparoscopic surgeons. Surg Endosc. 2010; 2588-2593.

6. Anton N, Montero P, Howley L, et al. "What stress-coping strategies are surgeons relying upon during surgery?” American Journal of Surgery 2015. E-publish.

7. Jennett, S. Churchill Livingstone's dictionary of sport and exercise science and medicine. Elsevier Health Sciences, 2008.

8. Williams, JM. Applied Sport Psychology: Personal Growth to Peak Performance. $6^{\text {th }}$ ed. New York: McGraw-Hill Higher Education, 2010.

9. Selder D, Burnett K, Nideffer R. Psychological factors associated with the successful completion of basic underwater demolition SEAL training. U.S. Navy Technical Report 1989.

10. McCrory, P, Cobley, S, Marchant, P. The effect of psychological skills training on selfregulation behavior, self efficacy, and psychological skill use in military pilot trainees. Military Psychology 2013;25:136-47. 
11. Le Scanff, C, Taugis, J. Stress management for police special forces. Journal of Applied Sport Psychology. 2002; 14:330-343.

12. Guenthner, SV, Hammermeister, J, Burton, D, Keller, L. Smoke and mirrors or wave of the future? Evaluating a mental skills training program for elite cross-country skiers. Journal of Sport and Behavior. 2010;33:3-24.

13. Clark, T, Williamon, A. Evaluation of a mental skills training program for musicians. Journal of Applied Sport Psychology 2011; 23:342-359.

14. Hoffman, SL, Hanraham, SJ. Mental skills for musicians: managing music performance anxiety and enhancing performance. Sport, Exercise, and Performance Psychology 2011;1:17-28.

15. Korndorffer, JR Jr, Dunne, JB, Sierra, R, et al. Simulator training for laparoscopic suturing using performance goals translates to the operating room. J Am Coll Surg 2005;201:23-9.

16. Stefanidis, D, Korndorffer, JR Jr, Markley, S, et al. Closing the gap in operative performance between novices and experts: does harder mean better for laparoscopic simulator training? J Am Coll Surg 2007;205:307-13.

17. Prabhu, A, Smith, W, Yurko, Y, et al. Increased stress levels may explain the incomplete transfer of simulator-acquired skill to the operating room. Surgery 2010;147:640-5.

18. Arora, S, Aggarwal, R, Moran, A, et al. Mental practice: effective stress management training for novice surgeons. J Am Coll Surg 2011b;212:225-33.

19. Wetzel, CM, George, A, Hanna, GB, et al. Stress Management Training for Surgeons-A Randomized, Controlled, Intervention Study. Ann Surg 2011;253:488-94.

20. Arora, S, Aggarwal, R, Sirimanna, P, et al. Mental practice enhances surgical technical skills: a randomized controlled study. Ann Surg 2011a;253:265-70. 
21. Immenroth, M, Bürger, T, Brenner, J, et al. Mental Training in Surgical Education. Ann Surg 2007;45: 385-91.

22. Arora, S, Aggarwal, R, Sevdalis, N, et al. Development and validation of mental practice as a training strategy for laparoscopic surgery. Surg Endosc 2010b;24:179-87.

23. Kern, DE, Thomas, PA, Hughes, MT. Curriculum Development for Medical Education : a Six-step Approach. 2nd ed. Baltimore, Md.: Johns Hopkins University Press, 2009. Print.

24. Society of American Gastrointestinal and Endoscopic Surgeons. Fundamentals of Laparoscopic Surgery (FLS). 2003-2008. Available at: http://www.flsprogram.org. Accessed February 23, 2015.

25. Peters JH, Fried GM, Swanstrom LL, et al. Development and validation of a comprehensive program of education and assessment of the basic fundamentals of laparoscopic surgery. Surgery 2004;135:21-7.

26. Hardy L, Roberts R, Thomas PR, Murphy, SM. “Test of Performance Strategies (TOPS): Instrument refinement using confirmatory factor analysis.” Psychology of Sport and Exercise 11.1 2010: 27-35.

27. Brickenkamp R, Zillmer E. The d2 test of attention. Hogrefe \& Huber Pub, 1998.

28. Spielberger, C.D. "State-Trait Anxiety Inventory." Corsini Encyclopedia of Psychology. 2010.

29. Tluczek A, Henriques JB, Brown RL. Support for the Reliability and Validity of a Six-Item State Anxiety Scale Derived From the State-Trait Anxiety Inventory. Journal of nursing measurement. 2009;17(1):19-28.

30. Arora, S, Aggarwal, R, Sevdalis, N, et al. Development and validation of mental practice as a training strategy for laparoscopic surgery. Surg Endosc 2010b;24:179-87. 
31. Hart S, Staveland L. Development of NASA-TLX (Task Load Index): Results of empirical and theoretical research. Advances in Psychology 1988; 52:139-183.

32. Ritter, EM, Scott, DJ. Design of a proficiency-based skills training curriculum for the fundamentals of laparoscopic surgery. Surgical Innovation 2007;14:107-12.

33. Hardy, L, Fazey, J. The inverted-U hypothesis: A catastrophe for sport psychology. University of Wales, Bangor, SHAPE, 1988.

34. Roscoe AH. Assessing pilot workload. Why measure heart rate, HRV and respiration? Biol Psychol 1992;34:259-87.

35. Veltman JA, Gaillard AW. Physiological workload reactions to increasing levels of task difficulty. Ergonomics 1998;41:656-69.

36. Varadhan KK, Neal KR, Dejong CH, Fearon KC, Ljungqvist O, Lobo DN. The enhanced recovery after surgery (ERAS) pathway for patients undergoing major elective open colorectal surgery: a meta-analysis of randomized controlled trials. Clin Nutr. 2010 Aug;29(4):434-40. 
Table 1. Mental Skills Curriculum: Modules, Goals, \& Objectives

\begin{tabular}{|c|c|c|c|}
\hline$\#$ & Module & Description & Goals \& Objectives \\
\hline $\mathbf{0}$ & $\begin{array}{l}\text { Overview and } \\
\text { Introduction } \\
\text { to MSC }\end{array}$ & $\begin{array}{l}\text { Overview of mental skills } \\
\text { training }\end{array}$ & $\begin{array}{l}\text { The goal of this session will be to introduce the } \\
\text { learner to the curriculum and reinforce the need for } \\
\text { mental skills training in surgical practice. By } \\
\text { participating in this session, the learner will... } \\
\text { - Briefly describe the history of Mental Skills } \\
\text { Training (MST) } \\
\text { - Report the rationale for MST in medicine } \\
\text { - Describe the process of the MSC }\end{array}$ \\
\hline 1 & $\begin{array}{l}\text { The Science of } \\
\text { Attention, } \\
\text { Focus, and } \\
\text { Concentration }\end{array}$ & $\begin{array}{l}\text { Overview of the science of } \\
\text { neurology and how it relates to } \\
\text { the skills in this curriculum }\end{array}$ & $\begin{array}{l}\text { The goal of this session will be to continue to } \\
\text { introduce the learner to the curriculum and teach } \\
\text { them the neurological science behind attention, } \\
\text { focus, and concentration. By participating in this } \\
\text { session, the learner will... Describe the differences } \\
\text { between bottom-up and top-down neurological } \\
\text { processes } \\
\text { - Review Nideffer's attentional model of } \\
\text { performance excellence }\end{array}$ \\
\hline 2 & Goal Setting & $\begin{array}{l}\text { Role of clear, effective goals in } \\
\text { achieving performance } \\
\text { excellence and building } \\
\text { confidence; importance of } \\
\text { clarifying both the tasks and } \\
\text { processes essential for success } \\
\text { in a procedure; includes } \\
\text { - Technical (e.g., nodal } \\
\text { points; clear performance } \\
\text { plan) } \\
\text { - Process (e.g., slow and } \\
\text { steady; breathe to remain } \\
\text { calm; optimal team } \\
\text { behavior) }\end{array}$ & $\begin{array}{l}\text { The goal of this session will be to equip the learner } \\
\text { with the knowledge and skills to establish clear and } \\
\text { effective performance goals. By participating in this } \\
\text { session, the learner will... } \\
\text { - Define and differentiate between outcome, } \\
\text { performance, and process goals } \\
\text { - Identify characteristics of his or her ideal } \\
\text { performance state } \\
\text { - Set technical goals for a procedure (for example, } \\
\text { identify 2-3 nodal points of a procedure) } \\
\text { - Identify process goals for a procedure (e.g., } \\
\text { staying calm, confident, and relaxed; optimal team } \\
\text { behavior) }\end{array}$ \\
\hline 3 & $\begin{array}{l}\text { Activation } \\
\text { Management }\end{array}$ & $\begin{array}{l}\text { Skills to relax physically and } \\
\text { mentally, as well as techniques } \\
\text { for raising energy level when } \\
\text { fatigued }\end{array}$ & $\begin{array}{l}\text { The goal of this session will be to equip the learner } \\
\text { with the knowledge and skills to manage physical } \\
\text { and mental states. By participating in this session, the } \\
\text { learner will... } \\
\text { - Demonstrate breathing and attention techniques to } \\
\text { achieve greater states of physical and mental } \\
\text { calm. (Sample Outcome: Reduce heart rate } 6 \text { beats } \\
\text { in } 12 \text { seconds) } \\
\text { - Demonstrate techniques to raise physiological }\end{array}$ \\
\hline
\end{tabular}




\begin{tabular}{|c|c|c|c|}
\hline & & & $\begin{array}{l}\text { activation and attention. (Sample Outcome: Raise } \\
\text { HR } 6 \text { beats in } 12 \text { seconds) }\end{array}$ \\
\hline 4 & $\begin{array}{l}\text { Attention } \\
\text { Management }\end{array}$ & $\begin{array}{l}\text { Techniques for maintaining } \\
\text { attention on what is essential } \\
\text { and ignoring distractions } \\
\text { - Thought stopping } \\
\text { - Self-talk } \\
\text { - Re-directing attention }\end{array}$ & $\begin{array}{l}\text { The goal of this session will be to equip the learner } \\
\text { with the knowledge and skills to effectively maintain } \\
\text { attention. By participating in this session, the learner } \\
\text { will... } \\
\text { - Identify personal negative self-talk in performance } \\
\text { situations and effective strategies for managing } \\
\text { self-talk } \\
\text { - Demonstrate ability to redirect attention from a } \\
\text { distraction to a target behavior }\end{array}$ \\
\hline 5 & Imagery & $\begin{array}{l}\text { Techniques for mental } \\
\text { rehearsal of both technical } \\
\text { aspects and non-technical skills } \\
\text { (e.g., managing emotions; } \\
\text { successfully dealing with } \\
\text { stressful events) }\end{array}$ & $\begin{array}{l}\text { The goal of this session will be to equip the learner } \\
\text { with the knowledge and skills to effectively mentally } \\
\text { rehearse. By participating in this session, the learner } \\
\text { will... } \\
\text { - Identify strategies for maximizing effective use of } \\
\text { imagery and mental rehearsal } \\
\text { - Incorporate imagery into practice and performance } \\
\text { situations }\end{array}$ \\
\hline 6 & $\begin{array}{l}\text { Refocusing } \\
\text { Strategies }\end{array}$ & $\begin{array}{l}\text { Techniques and principles for } \\
\text { handling various events that } \\
\text { can be stress inducing or } \\
\text { disruptive; learning how to } \\
\text { develop specific, } \\
\text { individualized strategies for } \\
\text { coping; plans address both } \\
\text { technical and non-technical } \\
\text { aspects of situation, including } \\
\text { team interactions }\end{array}$ & $\begin{array}{l}\text { The goal of this session will be to equip the learner } \\
\text { with the knowledge and skills to confront } \\
\text { challenging events in the OR. By participating in this } \\
\text { session, the learner will... } \\
\text { - Identify events which are particularly stress } \\
\text { inducing or distracting to the surgeon } \\
\text { - Devise personalized and specific strategies for } \\
\text { managing these situations }\end{array}$ \\
\hline 7 & $\begin{array}{l}\text { Preoperative } \\
\text { Mental } \\
\text { Routines }\end{array}$ & $\begin{array}{l}\text { Techniques and principles to } \\
\text { ensure that one is mentally } \\
\text { ready to perform, as well as } \\
\text { physically and technically } \\
\text { ready; includes preparation for } \\
\text { both the initiation of a } \\
\text { procedure and resuming after a } \\
\text { break or loss of focus }\end{array}$ & $\begin{array}{l}\text { The goal of this session will be to equip the learner } \\
\text { with the knowledge and skills to develop pre- } \\
\text { performance mental routines. By participating in this } \\
\text { session, the learner will... } \\
\text { - Develop a clear pre-performance "mental } \\
\text { readiness" routine for OR performance }\end{array}$ \\
\hline
\end{tabular}


Figure 1. d2 Test of Attention Sample

$$
\begin{aligned}
& \begin{array}{llllllllll}
\| & \| & \text { I } & \| & & & \text { II } & \text { I } & \| & \| \\
p & \text { d } & \text { p } & \text { p } & \text { d } & \text { d } & \text { d } & \text { d } & \text { p } & \text { d } \\
& & & \text { I } & \text { I } & \text { I } & \text { I } & & \text { I }
\end{array} \\
& \begin{array}{llllllllll}
\text { II } & \text { I } & & \text { II } & \text { I } & & \text { I } & & \text { I } & \\
\text { d } & d & d & \text { d } & p & p & d & p & d & p
\end{array}
\end{aligned}
$$

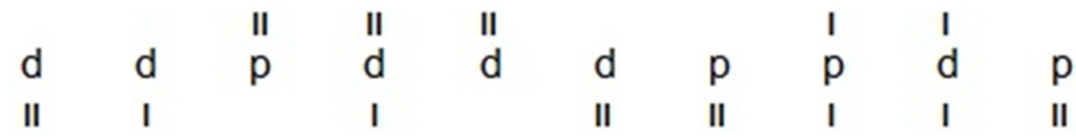


Figure 2. Effect of Training on Participant Mental Skills

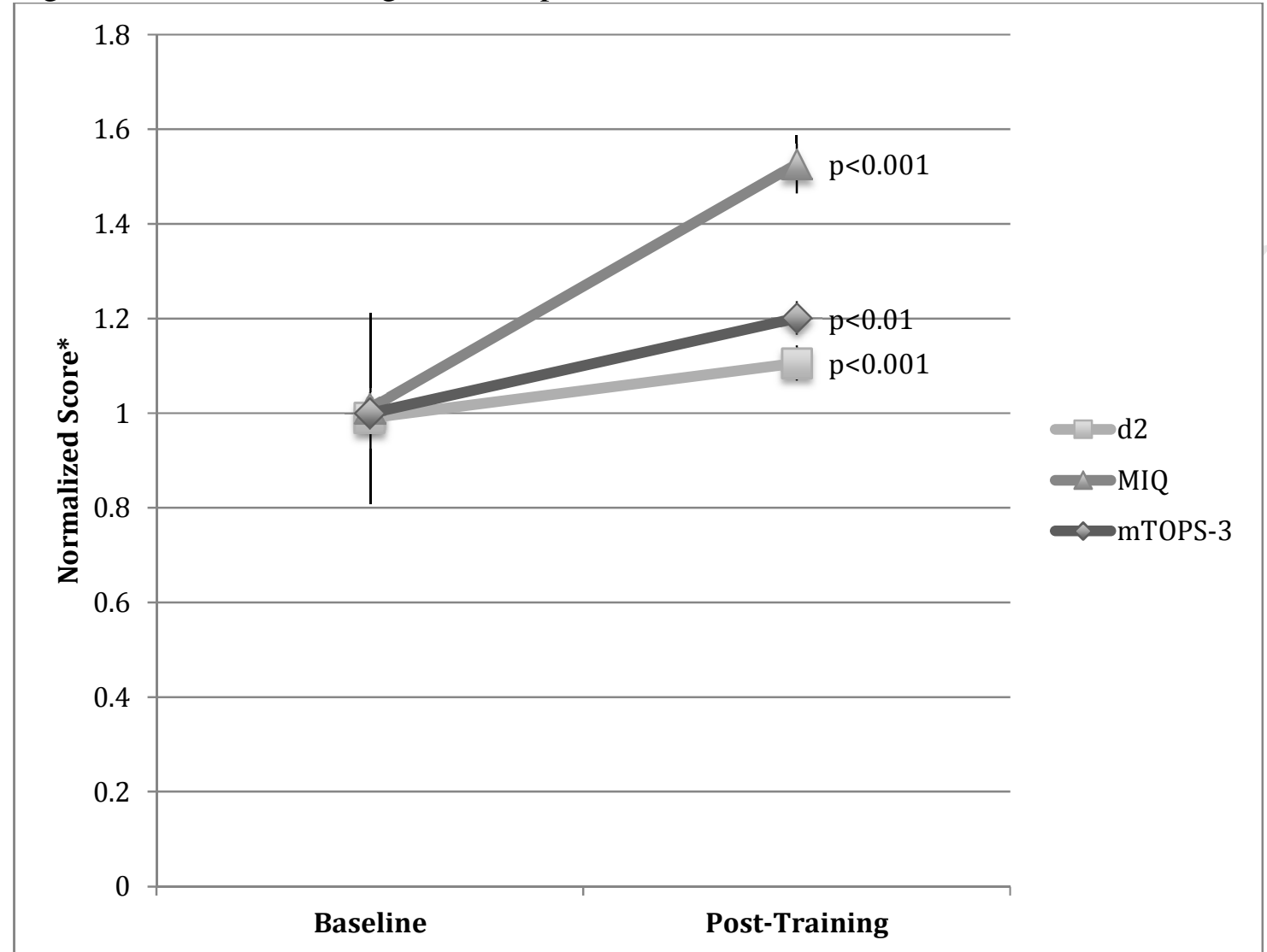

Higher scores indicate better attention (d2), mental imagery (MIQ), and psychological skill use and technique (mTOPS-3)

* Performance scores have been normalized to baseline 
Figure 3. Effect of training on participant stress and workload

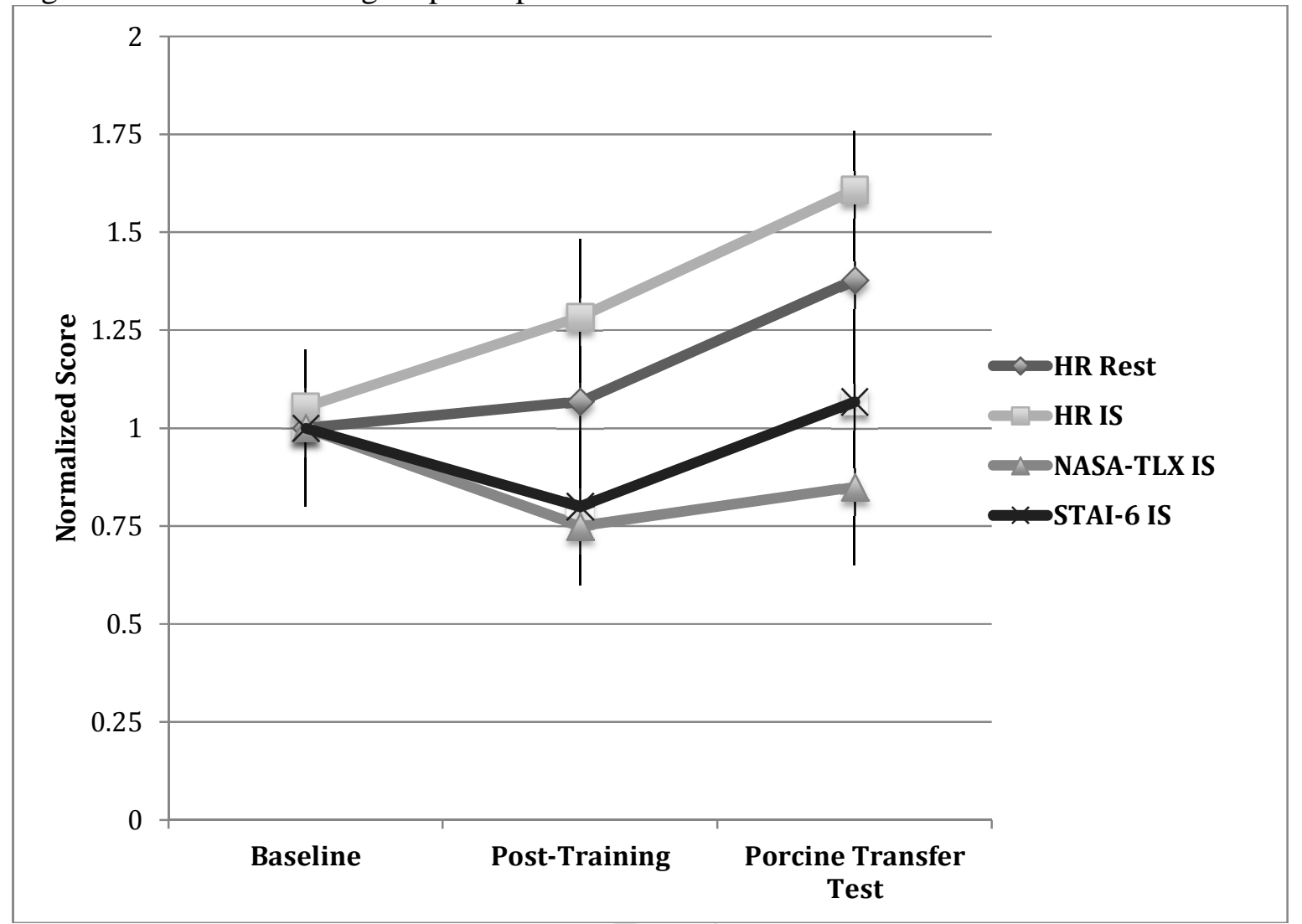

Lower scores indicate less physiological stress (HR), perceived anxiety (STAI-6), and workload (NASA-TLX). HR=heart rate, $\mathrm{IS}=$ intracorporeal suturing

* Performance scores have been normalized to baseline; all HR measurements have been normalized to resting HR at baseline 